\title{
John Herring (ed): Tachdjian's pediatric orthopaedics, 4th edn
}

\author{
Saunders Elsevier, 2007, 3 volume set with DVD Hardbound, \\ 3152 pp, ISBN-13: 978-1-4160-2221-3, ISBN-10: 1-4160-2221-X
}

\author{
Pierre H. Kehr • Claude Karger
}

Received: 11 June 2009 / Accepted: 30 June 2009 / Published online: 30 July 2009

(C) Springer-Verlag 2009

This new edition of Tachdjian's Pediatric Orthopaedics is presented in an outstanding and beautiful 3 volumes set, containing 2787 pages of text and illustrations, and including 2 DVDs providing filmed operative techniques for the most frequently used surgical procedures in children.

The first edition of this world famous textbook was published by Tachdjian in 1972. Since the beginning, the originality of this publication, compared to other books, was the addition of a large number of line drawings that illustrated the surgical procedures described in the text. The goal of this presentation was for the reader to know both the nature of the disease, its treatment choices, and in addition how to perform the surgery.

This new fourth edition, edited by John Herring of the Texas Scottish Rite Hospital, continues to follow the same spirit that inspired Dr Tachdjian, who died in 1996. The 3 volumes cover a large amount of knowledge in pediatric orthopaedics.

The text is presented in 43 chapters classified in to $6 \mathrm{sec}$ tions:

- Disciplines, covering general topics like growth and development, gait analysis, back pain, anesthesiology and management of the child with developmental disabilities

- Anatomic disorders, dealing with spine problems, disorders of the upper extremity, as well as hip, knee and foot anomalies

- Neuromuscular disorders, treating spinal and brain disorders and muscle diseases

P. H. Kehr $(\varangle) \cdot$ C. Karger

SOTEST, Strasbourg, France

e-mail: kehrpier@aol.com
- Orthopedic disorders, covering skeletal dysplasias and genetic syndromes, as well as metabolic, inflammatory and hematological conditions

- Musculoskeletal tumors, dealing with benign and malignant tumors of bone, cartilage and soft tissues

- Injuries, covering trauma of the spine, the upper and the lower extremities.

Each chapter is particularly well illustrated, including multiple color photographs of patients, imaging documents and drawings of surgical procedures. The color tables describing how to classify diseases, as well as multicolored drawings describing the surgical techniques are a significant improvement on the previous black and white line versions.

The reference list is extensive and quite encyclopedic.

The Editor has chosen not to attribute each chapter to his specific author, but to present the list of the 25 contributors at the beginning of the volumes. The chapters are therefore anonymous, and there is no way to know who wrote the text.

Since a long time, Tachdjian's Pediatric Orthopaedics has been considered as the "Bible" for medical educations in children's orthopedic care. This new edition is particularly user friendly to both the reader seeking a quick overview and the expert who needs to review all the current and pertinent information about a subject. Therefore, it is aimed to be useful for all the professionals involved in children's orthopedic problems: residents, fellows, staff surgeons, pediatricians, physiatrists and physical therapists.

Conflict of interest statement No funds were received in support of this study. 